\title{
Transport Signatures of Pseudomagnetic Landau Levels in Strained Graphene Ribbons
}

\author{
Diana A. Gradinar, ${ }^{1, *}$ Marcin Mucha-Kruczyński, ${ }^{1,2}$ Henning Schomerus, ${ }^{1}$ and Vladimir I. Fal'ko ${ }^{1}$ \\ ${ }^{1}$ Department of Physics, Lancaster University, LA1 4YB Lancaster, United Kingdom \\ ${ }^{2}$ Department of Physics, University of Bath, Claverton Down, Bath BA2 7AY, United Kingdom
}

(Received 13 March 2013; published 24 June 2013)

\begin{abstract}
In inhomogeneously strained graphene, low-energy electrons experience a valley-antisymmetric pseudomagnetic field which leads to the formation of localized states at the edge between the valence and conduction bands, understood in terms of peculiar $n=0$ pseudomagnetic Landau levels. Here we show that such states can manifest themselves as an isolated quadruplet of low-energy conductance resonances in a suspended stretched graphene ribbon, where clamping by the metallic contacts results in a strong inhomogeneity of strain near the ribbon ends.
\end{abstract}

DOI: 10.1103/PhysRevLett.110.266801

PACS numbers: 73.22.Pr, 62.20.-x, 71.70.Di

Graphene [1] is a one-atom-thick crystalline membrane [2] capable of withstanding reversible deformations of up to $10 \%$ [3], which is important because of the peculiar way strain affects the electronic properties of this material. It is a common feature of all materials with several degenerate valleys in the band structure [4] that the effect of their lattice deformations on electrons is equivalent to that of an effective gauge field $[3,5,6]$. In graphene, electrons near the Fermi level occupy states in the vicinity of a Dirac point (DP) at the edge between the valence and conduction bands, in one of two inequivalent valleys centered at the corners of the hexagonal Brillouin zone, $K$ and $K^{\prime}$. Consequently, inhomogeneous strain in graphene influences electron motion in a manner similar to an effective pseudomagnetic field, which has the opposite sign in the two valleys $[7,8]$. Recent scanning-tunneling experiments on graphene nanobubbles [9] have shown that even relatively weak deformations can generate pseudomagnetic fields that reach values equivalent to tens, or even hundreds, of Tesla, resulting in the formation of a discrete spectrum of Landau levels (LL), including the peculiar $n=0$ LL state pinned to the edge between the valence and conduction bands. Pseudomagnetic fields in deformed honeycomb crystals have also been engineered by an atomically controlled arrangement of $\mathrm{CO}$ molecules on a gold surface [10], and proposed in photonic analogues of graphene [11,12].

In contrast to the investigations of vertical transport in deformed graphene, there have been no reports yet on the observation of pseudomagnetic LLs in lateral transport studies. One can imagine that strained graphene nanoribbons (GNRs) would be a good candidate for this, since the deformations near the contact regions of clamped ribbons would be reproducibly inhomogeneous $[8,13]$. However, despite the vast literature, studies of the electronic transport in deformed GNRs mostly focused on homogeneously strained systems $[3,14,15]$ or on the interplay of inhomogeneous strain with real magnetic fields, and there is no theory showing how strain-induced localized states would manifest in the transport characteristics of GNRs. Here we show that pseudomagnetic $n=0 \mathrm{LL}$ states form in the contact regions of stretched GNRs and then give rise to a characteristic signature in the electronic transport: a quadruplet of low-energy conductance resonance, slightly split by the valley mixing and the tunnel coupling via evanescent modes in the middle part of the GNR.

The considered device is composed of a GNR that is clamped at the ends and contacted by heavily doped unstrained graphitic leads. We choose the ribbon to have armchair side edges along the transport direction $x$, and set contacts with bulk electrodes along the $y$ direction. Such a ribbon can be described using the tight-binding Hamiltonian [1]

$$
\mathcal{H}=\sum_{i} V_{i} c_{i}^{\dagger} c_{i}+\sum_{\langle i j\rangle} \gamma_{i j} c_{i}^{\dagger} c_{j},
$$

where $c_{i}$ is a fermionic annihilation operator acting on a site $i$ and $\langle i j\rangle$ denote pairs of nearest neighbors. Compared to pristine monolayer flakes, the on-site potential $V_{i}$ is modulated by strain, which we take into account by $V_{i}=(1 / 2) r\left(\partial \boldsymbol{\epsilon}_{c} / \partial r\right) \operatorname{div} \boldsymbol{u}\left(\boldsymbol{r}_{i}\right)$, where $\boldsymbol{u}=\left(u_{x}, u_{y}\right)$ is the displacement field of the membrane and $\epsilon_{c}$ is the on-site energy of electrons in a lattice with a given carbon-carbon bond length $r=1.42 \AA$. The hopping matrix elements,

$$
\gamma_{i j}=\gamma_{0} e^{\eta_{0}\left(l_{i j} / r-1\right)}, \quad l_{i j} \simeq r\left(1+\boldsymbol{n}_{i j} \cdot \hat{\boldsymbol{w}} \boldsymbol{n}_{i j}\right),
$$

depend on the distance $l_{i j}$ between lattice sites, modified by the strain [3]. Here $\hat{\boldsymbol{w}}$ is the $2 \times 2$ local strain tensor $w_{\alpha \beta}=1 / 2\left(\partial_{\alpha} u_{\beta}+\partial_{\beta} u_{\alpha}\right)$, with $\alpha, \beta=x$ or $y$, the parameter $\eta_{0}=\left(\partial \gamma_{0} / \partial r\right)\left(r / \gamma_{0}\right) \approx-3$ relates the change of the nearest neighbor coupling to the change of the bond length [16] $\left(\gamma_{0} \approx-3 \mathrm{eV}\right)$, and $\boldsymbol{n}_{i j}=(1,0),(-(1 / 2)$, $\sqrt{3} / 2)$ and $(-(1 / 2),-(\sqrt{3} / 2))$ are the unit vectors along carbon-carbon bonds in the honeycomb lattice. Clamping the ends of a suspended strained ribbon makes the strain distribution near the device contacts inhomogeneous [17], 
which we model using linear elasticity theory (see, e.g., Refs. [8,21,22]). The strain-induced asymmetry in the hoppings between neighboring carbon sites is equivalent

$$
e \mathcal{A}=\xi \frac{\hbar \eta_{0}}{2 r}\left(\begin{array}{c}
w_{x x}-w_{y y} \\
-2 w_{x y}
\end{array}\right)
$$

to the effect of a valley-dependent vector potential

(a)

(b)
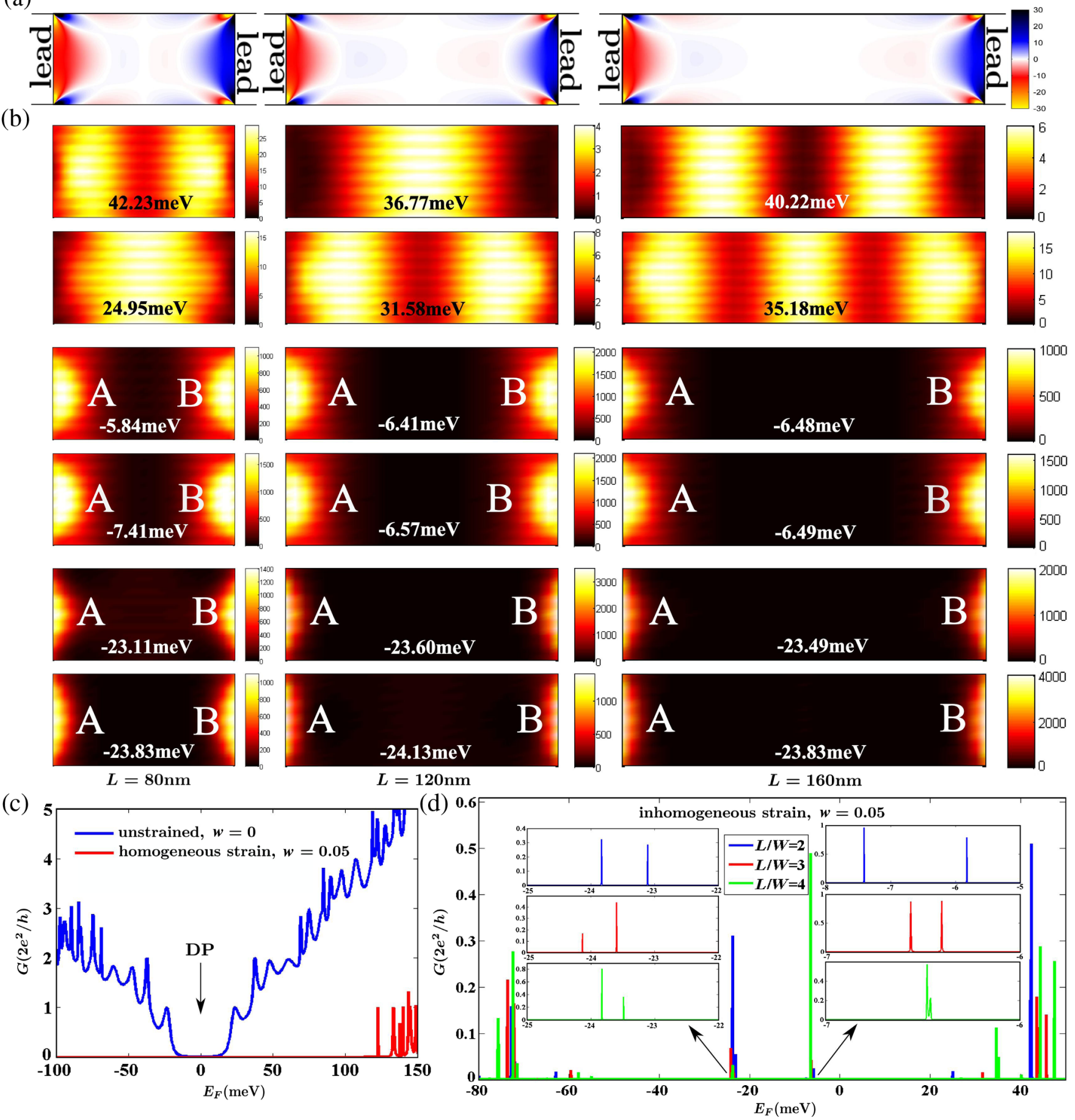

FIG. 1 (color online). We consider the transport through strained suspended graphene nanoribbons (GNR) which are clamped at highly doped contacts. (a) Distribution of pseudomagnetic fields $\mathcal{B}$ (in Tesla), defined according to Eq. (3), for electrons in the $K$ valley for GNR with $W \simeq 40 \mathrm{~nm}$ and aspect ratios $L / W=2,3$, and 4 . The inhomogeneous tensile strain in the middle of the nanoribbon is $w=0.05$. (b) Spatial structure of electron wave amplitudes corresponding to several resonances identified in (d), which displays the zero-temperature conductance of the ribbons as a function of the Fermi energy. For comparison, (c) shows the conductance for the ribbon with $L / W=3$ and no strain $(w=0$, blue) or artificially imposed homogeneous strain $(w=0.05$, red). 
where $\xi= \pm 1$ for valleys $K$ and $K^{\prime}$, which results in the appearance of a pseudomagnetic field $\mathcal{B}=\operatorname{rot} \mathcal{A}$ in the vicinity of the contacts $[7,8,22]$. This is illustrated in Fig. 1(a), for three inhomogeneously strained ribbons of width $W \simeq 40 \mathrm{~nm}$ and aspect ratio $L / W=2,3$, and 4, strained by $5 \%$ in their middle parts. The pseudomagnetic field is the largest positive (blue) or negative (red) near the contacts at the right and left ends, and is small in the middle of the ribbon.

The phase-coherent transport properties of two-terminal devices are encoded in the scattering matrix [23] which we evaluate using the recursive Green's function technique $[24,25]$, applied to the tight-binding model of the GNR sketched in Fig. 2. Within the Landauer-Büttiker formalism [26] we calculate the device conductance as a function of the Fermi level in the middle part of the GNR, for a given height $(V=-200 \mathrm{meV})$ of the gate-controlled potential energy step between the doped graphene leads and the suspended part. The resulting device is an $n-p-n$ $\left(E_{F}<0 \mathrm{meV}\right)$ or $n-n^{\prime}-n\left(E_{F}>0 \mathrm{meV}\right)$ graphene junction, where most of the conductance features are determined by scattering from the strain-modified $n-p$ or $n-n^{\prime}$ interfaces.

Homogeneous strain.-Figure 1(c) shows the Fermienergy dependence of the zero-temperature conductance of the ribbon with $L / W=3$, for no strain (blue) and for artificially imposed 5\% homogeneous strain (red). The unstrained armchair GNR is semiconducting; the conductance therefore exhibits a gap around the DP $\left(E_{F}=\right.$ $0 \mathrm{meV})$. The conductance oscillations away from the DP are due to the Fabry-Pérot-like standing wave resonances in the electron transmission across the potential barrier geometry [27]. For a homogeneously strained armchair

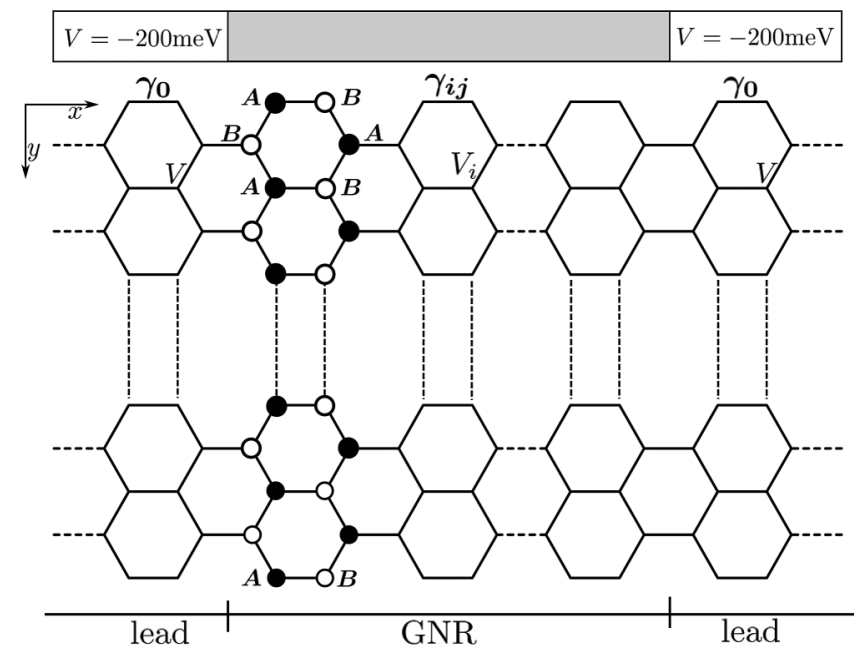

FIG. 2. Sketch of the tight-binding model (1) of the GNR junction with armchair boundaries. The system is composed of two ideal heavily doped leads $(V=-200 \mathrm{meV})$ and a central suspended region, in which strain modulates the hopping matrix elements $\gamma_{i j}$ and the on-site energy $V_{i}$.
GNR, $w_{x x}=w, w_{y y}=-\sigma w$, and $w_{x y}=0$, where $\sigma=$ 0.165 is the Poisson ratio for graphite [28] and $w$ parametrizes tensile strain. For $w=0.05$ homogeneous strain the conductance is completely suppressed for $\left|E_{F}\right|<$ $100 \mathrm{meV}$. This is because the constant vector potential induced by the homogeneous strain shifts the Dirac cones away from the $K$ and $K^{\prime}$ corners of the Brillouin zone, perpendicular to the transport direction [3]. The misalignment between the Fermi surfaces in the unstrained leads and in the strained suspended region results in a suppression of the conductance in the ballistic regime [15]. The threshold for such an insulating behavior for parameters used in Fig. 1(c) is $w=0.024$, and can be lowered by reducing the height of the potential step $V$ between the central part of the ribbon and the contacts.

Inhomogeneous strain.-This behavior is significantly changed when the inhomogeneity of the strain is taken into account. Figure 1(d) shows the Fermi-energy dependence of the conductance for the inhomogeneously strained ribbons shown in Fig. 1(a). In contrast to Fig. 1(c), here we find several groups of additional sharp resonance conductance peaks in the energy range $\left|E_{F}\right|<100 \mathrm{meV}$. To reveal the nature of each group of these peaks, we analyze the spatial distribution of the corresponding electronic states. Within the Landauer-Büttiker formalism, this can be obtained from the response to local perturbations of the scattering amplitudes at the energies close to the resonance conditions [29]. The reconstructed spatial structure of the resonance states is shown in Fig. 1(b). As illustrated in the top two rows, the states away from the DP correspond to Fabry-Pérot-like standing waves formed in the homogeneously strained central part of the structure, to which they are confined due to electron reflection from the interfaces. These resonances appear because the transverse momentum is no longer conserved when the interface region is inhomogeneous, which allows carriers to overcome the misalignment between the Fermi surfaces described above. However, two groups of resonances, in the energy range $-25<E_{F}<0 \mathrm{meV}$ just below the DP, display a very different behavior. As shown in the insets in Fig. 1(d), these resonances occur in almost degenerate pairs, of which we find two groups. The spatial structure of these states, shown in the bottom four rows of Fig. 1(b), clearly resembles the pseudomagnetic field distribution [17].

We now demonstrate that this quadruplet of resonances can be attributed to the $n=0$ pseudomagnetic Landau level. Our main piece of evidence is a unique feature of this LL in graphene, namely, that the electron amplitude resides on either the $A$ or $B$ sublattice. The selected sublattice depends on the sign of $\mathcal{B}$ but is independent of the valley [5]. In contrast, higher LLs and Fabry-Pérot-like resonances occupy both sublattices equally. By selectively placing the probing perturbation on the $A$ or $B$ sites, we find, as illustrated for one example in Fig. 3, that the low-energy resonances have the following property: their 


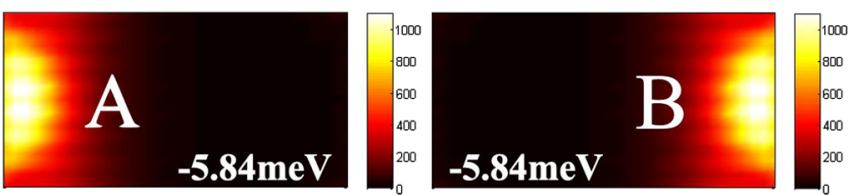

FIG. 3 (color online). Sublattice-resolved electron amplitude for one of the resonances in Fig. 1(d) $(L / W=2, E=$ $-5.84 \mathrm{meV}$ ), obtained by placing the probing perturbation on the A (left) or B (right) sites.

amplitude is high on the $A$ sites near the left end of the ribbon (where $\mathcal{B}<0$ ), and the amplitude is high on the $B$ sites near the right end (where $\mathcal{B}>0$ ). This fully agrees with the unique feature of the $n=0$ LL described above.

The fact that we find four such low-energy resonances, as well as the dependence of their splitting on the length of the ribbon, further supports this interpretation of the origin of these states. The $y \rightarrow-y$ reflection symmetry maps valleys $K$ and $K^{\prime}$ onto each other. The resulting symmetric and antisymmetric superpositions of the two valley manifestations of the $n=0$ LL lead to a splitting of the quadruplet into two groups: a low-energy branch at $E_{F} \approx-24 \mathrm{meV}$, which is valley symmetric and displays a maximum on the symmetry axis, and a high-energy branch at $E_{F} \approx-7 \mathrm{meV}$, which displays a nodal line on this axis. Each group splits further into two narrowly spaced lines because of the tunnel coupling of the states $\Psi_{L}^{0}$ and $\Psi_{R}^{0}$ near the left and right ends of the ribbon, which is provided by the evanescent tails of the electronic wave functions in the middle part, where $\mathcal{B}$ is small. Note that the splitting in each of these pairs is smaller in a longer ribbon, since the overlap of the evanescent tails of $\Psi_{L}^{0}$ and $\Psi_{R}^{0}$ decreases with the separation between the GNR ends.

In conclusion, we describe a unique transport signature of the pseudomagnetic field in a strained suspended graphene nanoribbon, namely, the resonant transmission via the sublattice-polarized $n=0$ pseudomagnetic Landau level. These states form near the inhomogeneously strained contact regions and give rise to a characteristic quadruplet of conductance resonances near the Dirac point. The above-proposed analysis is directly applicable to graphene ribbons where high-quality armchair edges are obtained by the oriented growth on patterned $\mathrm{SiC}$ substrates [30], etching of graphene samples with catalytic nanoparticles [31], or chemical derivation [32]. Even though imperfections in the system will lead to the appearance of additional conductance resonances, the $n=0$ Landau level state is protected against the influence of disorder by its unique position at the energy of the Dirac point and its energetic separation from the Fabry-Pérot-like resonances.

We thank A. Geim and H. Ochoa for useful discussions. This project was funded by EC STREP ConceptGraphene, EPSRC S\&IA grant, ERC Advanced Grant Graphene and Beyond, and by the Royal Society Wolfson Research Merit Award. *d.cosma@lancaster.ac.uk

[1] A. H. C. Neto, F. Guinea, N. M. R. Peres, K. S. Novoselov, and A. K. Geim, Rev. Mod. Phys. 81, 109 (2009).

[2] C. Lee, X. Wei, J. W. Kysar, and J. Hone, Science 321, 385 (2008); T. J. Booth, P. Blake, R. R. Nair, D. Jiang, E. W. Hill, U. Bangert, A. Bleloch, M. Gass, K. S. Novoselov, M. I. Katsnelson, and A. K. Geim, Nano Lett. 8, 2442 (2008); J.S. Bunch, S.S. Verbridge, J.S. Alden, A. M. van der Zande, J.M. Parpia, H. G. Craighead, and P.L. McEuen, ibid. 8, 2458 (2008).

[3] V. M. Pereira, A. H.C. Neto, and N.M. R. Peres, Phys. Rev. B 80, 045401 (2009); R. M. Ribeiro, V. M. Pereira, N. M. R. Peres, P. R. Briddon, and A.H.C. Neto, New J. Phys. 11, 115002 (2009).

[4] S. V. Iordanskii and A. E. Koshelev, JETP Lett. 41, 574 (1985).

[5] F. Guinea, M. I. Katsnelson, and A. K. Geim, Nat. Phys. 6, 30 (2010); F. Guinea, A. K. Geim, M. I. Katsnelson, and K. S. Novoselov, Phys. Rev. B 81, 035408 (2010).

[6] M. A. H. Vozmediano, M. I. Katsnelson, and F. Guinea, Phys. Rep. 496, 109 (2010); D. Rainis, F. Taddei, M. Polini, G. León, F. Guinea, and V. I. Falko, Phys. Rev. B 83, 165403 (2011).

[7] H. Suzuura and T. Ando, Phys. Rev. B 65, 235412 (2002); J. L. Mañes, Phys. Rev. B 76, 045430 (2007).

[8] E. Prada, P. San-Jose, G. León, M. M. Fogler, and F. Guinea, Phys. Rev. B 81, 161402 (2010).

[9] N. Levy, S. A. Burke, K. L. Meaker, M. Panlasigui, A. Zettl, F. Guinea, A.H.C. Neto, and M.F. Crommie, Science 329, 544 (2010).

[10] K. K. Gomes, W. Mar, W. Ko, F. Guinea, and H. C. Manoharan, Nature (London) 483, 306 (2012).

[11] H. Schomerus and N. Y. Halpern, Phys. Rev. Lett. 110, 013903 (2013).

[12] M. C. Rechtsman, J. M. Zeuner, A. Tünnermann, S. Nolte, M. Segev, and A. Szameit, Nat. Photonics 7, 153 (2013).

[13] W. Bao, F. Miao, Z. Chen, H. Zhang, W. Jang, C. Dames, and C. N. Lau, Nat. Nanotechnol. 4, 562 (2009); M.L. Teague, A. P. Lai, J. Velasco, C. R. Hughes, A. D. Beyer, M. W. Bockrath, C. N. Lau, and N.-C. Yeh, Nano Lett. 9, 2542 (2009); K. S. Kim, Y. Zhao, H. Jang, S. Y. Lee, J. M. Kim, K. S. Kim, J.-H. Ahn, P. Kim, J.-Y. Choi, and B. H. Hong, Nature (London) 457, 706 (2009).

[14] Y. Li, X. Jiang, Z. Liu, and Z. Liu, Nano Res. 3, 545 (2010); Y. Lu and J. Guo, Nano Res. 3, 189 (2010).

[15] V. M. Pereira and A.H.C. Neto, Phys. Rev. Lett. 103, 046801 (2009); M. M. Fogler, F. Guinea, and M.I. Katsnelson, Phys. Rev. Lett. 101, 226804 (2008).

[16] R. Ferone, J. R. Wallbank, V. Zolyomi, E. McCann, and V. I. Fal'ko, Solid State Commun. 151, 1071 (2011).

[17] Note that we have neglected the possibility of spontaneous wrinkling [18] since strain limits their formation in suspended samples by increasing the transverse rigidity [19]. We have also applied a finite cutoff to regularize the formally divergent displacements [20] one finds using linear elasticity theory [21] near the corners of clamped ends of the ribbon. This regularization accounts for the breakdown of linear elasticity theory due to nonlinearities. We note that the states in Fig. 1 are localized away from the corners, and thus are insensitive to the precise value of the imposed cutoff. 
[18] E. Cerda and L. Mahadevan, Phys. Rev. Lett. 90, 074302 (2003).

[19] E. V. Castro, H. Ochoa, M. I. Katsnelson, R. V. Gorbachev, D. C. Elias, K. S. Novoselov, A. K. Geim, and F. Guinea, Phys. Rev. Lett. 105, 266601 (2010).

[20] M. L. Williams, J. Appl. Mech. Trans. 19, 526 (1952).

[21] S.P. Timoshenko and J. N. Goodier, Theory of Elasticity (McGraw-Hill, Singapore, 1970).

[22] M. Mucha-Kruczynski and V.I. Fal'ko, Solid State Commun. 152, 1442 (2012).

[23] C. W. J. Beenakker, Rev. Mod. Phys. 69, 731 (1997).

[24] S. Datta, Electronic Transport in Mesoscopic Systems (Cambridge University Press, Cambridge, England, 1988).

[25] J. P. Robinson and H. Schomerus, Phys. Rev. B 76, 115430 (2007).

[26] M. Büttiker, Phys. Rev. Lett. 57, 1761 (1986).
[27] M. I. Katsnelson, K. S. Novoselov, and A. K. Geim, Nat. Phys. 2, 620 (2006); T. Tudorovskiy, K. J. A. Reijnders, and M.I. Katsnelson, Phys. Scr. T146, 014010 (2012).

[28] O. L. Blakslee, D. G. Proctor, E. J. Seldin, G. B. Spence, and T. Weng, J. Appl. Phys. 41, 3373 (1970).

[29] V. Gasparian, T. Christen, and M. Büttiker, Phys. Rev. A 54, 4022 (1996).

[30] J. Hicks, A. Tejeda, A. Taleb-Ibrahimi, M. S. Nevius, F. Wang, K. Shepperd, J. Palmer, F. Bertran, P. Le Fèvre, J. Kunc, W. A. de Heer, C. Berger, and E. H. Conrad, Nat. Phys. 9, 49 (2012).

[31] L. C. Campos, V. R. Manfrinato, J. D. Sanchez-Yamagishi, J. Kong, and P. Jarillo-Herrero, Nano Lett. 9, 2600 (2009).

[32] X. Li, X. Wang, L. Zhang, S. Lee, and H. Dai, Science 319, 1229 (2008). 